Marquette University

e-Publications@Marquette

English Faculty Research and Publications

English Department

7-23-2017

Intuition in Healthcare Communication Practices: Initial Findings from a Qualitative Inquiry

Elizabeth L. Angeli

Marquette University, elizabeth.angeli@marquette.edu

Lillian Campbell

Marquette University, lillian.campbell@marquette.edu

Accepted version. 2017 IEEE International Professional Communication Conference (ProComm), (July 23-26, 2017) DOI. (C) 2017 IEEE. Used with permission. 


\title{
Intuition in Healthcare Communication Practices: Initial Findings from a Qualitative Inquiry
}

\author{
This is an Accepted Manuscript of an article published by IEEE Explore Digital Library in
}

IEEE International Professional Communication Conference Proceedings available online:

http://ieeexplore.ieee.org/Xplore/home.jsp

\author{
Elizabeth L. Angeli \\ Marquette University \\ Lillian Campbell \\ Marquette University \\ elizabeth.angeli@marquette.edu lillian.campbell@marquette.edu
}

\begin{abstract}
This brief paper reports on how healthcare providers negotiate stages of care and communication by using intuition. This focus shifts attention away from the product - patient records-and towards the process of medical communication. To support this claim, the paper presents preliminary findings from qualitative analysis of two individual ethnographic research projects with live-action clinical nursing simulations and emergency medical services. Using a grounded theory analysis that identified intuitive moments in the writing practices of healthcare providers, this brief paper demonstrates how intuition manifests in all five stages of care-anticipate, assess, plan, act and reassess, and document-and grounds medical assessment and decision making. Analysis suggests three takeaways for healthcare communicators and educators: 1. intuitive work supports patient specific and responsive care; 2. coding and highlighting mediate patient sense; and 3. recognizing and valuing patient sense is a learned skill. The paper concludes with suggestions for reflective activities that could support incorporating intuition into healthcare communication pedagogy.
\end{abstract}

Index Terms - Intuition, writing, healthcare, communication

\section{INTRODUCTION}

In the age of evidence-based medicine, medical decision-making is based on data and improved outcomes supported by quantitative information. But not all medical decisions can be buoyed by data alone; some medical decisions rely on gut feelings and experience [1], [2], [3]. The challenge, though, is that experience is not quantifiable and is linked with a seemingly unconscious force: intuition. In this brief paper, we argue that healthcare providers' intuition plays a role as they negotiate stages of care and communication.

To illustrate this intuitive work, we draw on two ethnographic research studies with nursing simulations and emergency medical services (EMS) professionals. By focusing on the intuitive work behind communication, we highlight the complexity of communication; instead of looking at the product - patient records - the process unfolds, suggesting ways to gain a deeper understanding of medical communication in different environments.

Legally binding documents, like the patient health record, prompt nursing students and EMS professionals to support decision-making processes with detailed evidence. This evidence, though, is not always tangible and can manifest instead as intuitive knowledge. Intuition refers to using "experience to recognize key patterns that indicate the dynamics of the situation" [4]. In our qualitative data, participants often referenced "gut feeling" or "experience," mentioning that these moments influenced patient care and documentation. Thus, drawing on theories from technical communication and findings from qualitative analysis, we consider how providers communicate intuitive information in written and verbal genres that are designed to de-emphasize non-scientific information. We end by suggesting how healthcare educators can prepare students for this communicative practice. 


\section{GUIDING THEORETICAL FRAMEWORKS}

Technical communication scholars have studied how intangible elements, like cognition, facilitate the workplace writing process, allowing researchers to understand how writers interact with their surrounding environment, how writers draw on memories and integrate them into writing, and how writers rely on external cues to facilitate that process [5], [6], [7], [8]. Other fields have also proposed methods of studying intuition and decision making, including psychology [9], [10]. However, their approaches do not address the communication practices that occur alongside intuitive action. Building on these approaches, our project also draws on work in technical communication that unpacks the relationship between embodied immersion in professional contexts and writing practices.

Medical professionals' knowledge parallels what Sauer dubbed in her research on miners' embodied sensory knowledge as "pit sense . . . direct physical sensations felt or perceived in highly specific local environments" [11]. In Sauer's research, pit sense is not accounted for in technical documents because miners do not "record their reactions in written communication" [12]. For our purposes, we use the term "patient sense" to describe the embodied sensory knowledge that medical providers gain from physical presence in the patient's environment and in direct contact with the patient. Patient sense can include a range of intuitive, physical experiences in the room with the patient such as a certain kind of smell, a physical feature that does not look or feel quite right, or an off-hand comment that is an indicator of a larger problem.

Sauer also argues that two additional modes of knowing-experience, which is "embodied in objects and materials" and scientific knowledge, which is "sensed or perceived as data in language, physical tracings, and inscriptions"-guide workplace communication [13]. In the workplace, medical professionals rely on professional experience embodied in objects and materials that mediate patient interactions, such as the stethoscope and the thermometer. And they rely on "medical knowledge," e.g., the patient data that comes to them from telemetry machines and the reports received from ultra-sounds or blood tests. The boundaries between these different modes of knowing are not easily drawn but all three come into play as medical providers negotiate the process of organizing and translating patient information.

Building on these modes of knowing and Sauer's concept of "pit sense," we investigate how nurses and EMS professionals transcribe their intuition and patient sense so that it is legible to other health care providers. We argue that participants followed Goodwin's concept of "professional vision" by coding, which "transforms phenomena observed in a specific setting into the objects of knowledge," and highlighting, which "makes specific phenomena ... salient by marking them in some fashion" [14], [15].

For example, if a medical professional begins to have concerns about domestic abuse gathered from observing unusual bruises on a woman's body and interactions with her spouse, there is unlikely to be a drop-down menu in the patient's chart to easily capture those intuitive concerns. However, the professional will likely work to "code" those concerns into the chart by documenting the bruising and including a note about the patient's reaction to her husband. These details might be highlighted during a handoff conversation in which the provider calls attention to those particular notes in the chart over others. Coding and highlighting, then, offer two concepts for understanding how medical professionals communicate their patient sense to other providers, both verbally and textually.

\section{METHODS}

The findings in this paper are based on qualitative analysis of data from two individual ethnographic research projects with live-action nursing clinical simulations and EMS.

Clinical nursing simulations are widely used to train undergraduate and graduate nursing students in technical practice and communication. In the simulations that were the subject of this research, junior year nursing students were immersed in a pre-written patient scenario and asked to collaboratively provide care (in teams of 2-3 students) for a robotic patient as its condition worsened. Activities included negotiating a plan for care based on the physician's orders, administering medications and tracking vital signs, and documenting interventions. The simulation project drew on a year of qualitative research including field observations of simulations and debrief conversations, focal student interviews, video recordings of simulations, and collection of a range of texts including patient charts.

The EMS project included 16 months of participant observation, interviews, and surveys to better understand the shifting environments in which EMS professionals write. Fifteen participants completed surveys, and 12 participants completed interviews. Before this study began, the researcher was enrolled in an EMT-Basic course and earned her EMT-B certification during the study's duration. As an EMT-B student, she completed a 7-month course and clinical rotations in the emergency department and on ambulances where she cared for patients. Data included field notes from participant observation in the emergency room and on the ambulances, completed surveys, interview transcripts, and templates of patient care report forms on which participants documented patient care.

In both research projects, participants managed vast amounts of information in new environments: nursing 
students were new to the field of nursing and the simulation context, and EMS professionals enter new environments daily when they respond to a 911 call.

Data analysis began with identifying excerpts in field notes and interviews from both projects where intuition was discussed or visible in care. After identifying these intuitive moments, we used a grounded theory approach to code for patterns across examples [16], [17]. Ultimately, categorizing excerpts by the stage of care helped make visible how practitioners use intuition to manage their environments, care for patients, and communicate their findings to other providers.

\section{EXTENDED EXAMPLES AND ANALYSES}

Our preliminary analysis suggests that intuition complements evidence-based data, like observations, vital signs, or documentation from other healthcare providers. Because of its intangible nature, intuitive data is harder to identify in transcripts and field notes, but we noted how participants referred to aspects of intuition-experience, trust, and gut feelings - to guide their actions and communication while completing stages of patient care: anticipate, assess, plan, act and reassess, and document.

The stages of patient care may differ across medical specialties, but at their core, they generally include five broad steps. First, participants in our projects surveyed a patient's history or the patient's environment to obtain a general picture of the patient's chief complaint; in doing so they began to anticipate what care the patient will need. Next, using this information, participants assessed a patient's condition to determine-plan-treatment (participants did not formally diagnose patients because it was beyond their scope of care). After planning treatment, participants acted on their plan and reassessed the effectiveness of these plans, adjusting where needed. Then, they documented treatment plans and information that guided decision making throughout these stages of care.

\section{Anticipate}

In both research projects, participants demonstrated that they relied on previous learning and experience to help them anticipate a patient's needs and corresponding treatment plans. Information from other communicators involved in a response, like dispatch for EMS or a previous nursing team for nurses, offered participants pertinent information that cued them into expectations when meeting a patient.

In a clinical nursing simulation, Michelle was part of an incoming team preparing to care for a diabetic geriatric patient who recently had surgery. During the handoff of patient care between nursing teams, the outgoing group highlighted verbally and in their documentation the risk that the patient's urine would soak a newly changed wound dressing. They also attempted to code a more general concern for the patient's well-being in conversation: "how conscious she is and stuff . . make sure she's good." Following up on this comment, Michelle asked, "Have you guys taken her blood sugar after [eating] since she's been here?" With knowledge that the patient is diabetic, Michelle drew on intuition about patient needs that occurred even prior to physical or verbal interaction.

In her interview, Michelle attributed this question to her experiences in coursework, saying: "We've been talking a lot about like problematic illnesses and stuff in geriatric care." This example demonstrates how anticipation of patient needs emerges at an intersection between the previous providers' coding and highlighting of their patient sense and the participant's previous experiences and learning.

\section{Assess}

Once participants anticipated a patient's needs based on another provider's coding and highlighting, they began to assess the patient's condition. When assessing a patient, participants combined their experience and "gut feeling" to corroborate information with the "anticipate" stage.

For example, one veteran paramedic offered the example of being dispatched and responding to a patient who "was supposedly having an allergic reaction to Febreze"; when they arrived to the patient's home, he noted that he would grow "suspicious" if he heard people "shuffling around" upstairs, hiding from the first responders, suggesting that this patient may not be having an allergic reaction but instead is in a drug-induced state. This skill, he noted, comes with experience, "Learning to listen to your gut, learning to know, 'Okay, gee, I've been in this situation before ...",

By referring to previous experience at this stage of patient care, participants' responses indicate that the assessment stage draws on intuition as they decideassess-which information is important to remember. Intuitive knowledge, like the sense that people may be hiding, becomes recognizable to providers as they observe their surroundings and integrate that sensory information with previous experience, marking a "gut feeling" about a situation. They then have to decide both how to act upon that patient sense and how to code and highlight it for communication to other health professionals.

\section{Plan}

In the plan stage, participants combined previously collected information with intuition to choose the best course of action for a patient. One paramedic noted that planning was part of the art of "practicing medicine," which includes seeking information by asking the patient and other key personnel questions, drawing on professional and previous experience, and trusting that experience to choose an appropriate treatment plan. This 
aligns with Klein's definition of intuition, the ability to recognize patterns in a situation.

In the contexts of nursing simulations and EMS, key patterns include patient behavior, environmental cues, patient history, and a patient's signs and symptoms. By seeking out this information, participants identified patterns that enabled them to code intuitive knowledge into a recognizable course of action-a treatment decision.

The challenge, as participants noted, is trusting experience and gut feeling-intuition-at this stage, indicating that treatment decisions cannot be guided by textbook knowledge alone but rely on intangible, intuitive work.

\section{Act and Reassess}

After using intuition to plan treatment, participants acted on their plans and re-assessed a patient's condition to ensure the chosen plan effectively treated the patient. At this stage, participants' abilities to "read" a patient or an environment facilitated action, and this patient sense drew on previous experience and attuning to "red flags."

For example, when working with a patient complaining of pain, an EMS participant noted that the patient asked for morphine as soon as he was loaded onto the ambulance on a stretcher. Based on this participant's experience, patients do not ask for morphine unless they have already used it and might be seeking the drug by calling 911. This participant did not give the patient morphine because he "read him better than that," suggesting that the participant's experience helped him code intuitive knowledge into a professional course of action throughout the response. In this case, that course of action was a decision to withhold pain medication while transporting a patient to the hospital after the anticipate, assess, and plan stages. This participant's patient sense developed as the patient spoke, leading the participant to a treatment decision that was based on intuitive knowledge.

\section{Document}

In the document phase, participants captured and translated their intuition about a patient's condition for future providers. In doing so, their documentation supported the incoming group in intuiting and anticipating patient needs.

For example, in a clinical nursing simulation, a team of students located a warm protrusion on a patient's leg and ordered an ultrasound to confirm a blood clot. This team did not receive doctor confirmation before their shift ended, so they needed to capture their intuition about the leg's condition without diagnosing a clot. The team documented that they took off the Sequential Compression Device (SCD) in response to the patient's complaints of leg pain: "SCD's off: Sharp/stabbing pain Left calf." Under "Hand-off Report," they documented:
"Left leg: edema, pain, no pedal pulse, provider called, will bring new orders." By emphasizing pain, lack of pulse, and swelling, the team coded their concerns for an incoming group, working to capture their intuition-their gut feeling - about the leg's condition. A student, Ryan, who was part of the incoming team noted his burgeoning ability to interpret this coding of patient sense, reflecting, "I can look at the board and see like, 'Oh they had calf pain and they also grouped that it was swollen at the same time. I think they're thinking a DVT [Deep Vein Thrombosis], you know I'm also thinking a DVT.",

\section{DISCUSSION}

The above analysis suggests three takeaways for healthcare communicators and healthcare educators: 1 . intuitive work supports patient specific and responsive care; 2. coding and highlighting mediate patient sense; and 3. recognizing and valuing patient sense is a learned skill.

First, no two patients are alike and care plans reflect and respond to patient needs. As such, participants came to each patient with a general idea of what to expect in the anticipate stage based on communicated information and their own experience and training. As participants interacted more with specific patients and their intuition developed, they selected treatment plans, provided treatment, and reassessed these decisions. This ensured that intuition - coupled with other types of evidence, like vital signs-guided participants to responsive treatment decisions.

Second, most of the stages of patient care involve coding patient sense, either internally as providers interpret their feelings about a patient through the lens of a defined treatment plan or collaboratively as they communicate their sense of patient experience to other providers. In the last stage, "Document," participants supported other healthcare providers by coding and highlighting their patient sense in verbal exchanges and patient health records, despite the lack of official space in these records to document their intuitive work. Thus, intuition complements the visible, communicated work of healthcare, mainly the data written in patient health records and spoken to other healthcare providers.

Finally, recognition and integration of patient sense into all stages of care is a learned skill, one that is just as important as tangible skills like inserting a Foley catheter. Participants in both contexts emphasized that their ability to leverage intuition to guide care was something that developed over time and through multiple successes and failures. By attuning to their embodied feelings and internalizing previous experience, they learned to trust their patient sense and to value it at every stage of patient care. This need to attune to and trust intuition raises the question of how instructors might scaffold for integration of intuitive work in health communication pedagogy. 


\section{CONCLUSION AND PEDAGOGICAL IMPLICATIONS}

This brief paper suggests that as healthcare providers learn how to assess patients and communicate with others they also remain mindful of their intuition: how it develops and how it guides them as they practice medicine. Instructors will often acknowledge intuition's role in patient care when teaching; however, because the documents that guide healthcare communication prioritize concrete, quantitative evidence, intuition is rarely emphasized in the teaching of healthcare communication practices.

Because written and verbal reflection is already a mainstay in pedagogy for healthcare providers [18], [19], [20], reflective activities provide a natural opportunity to scaffold awareness about the relationship between intuition and writing. Reflective activities might follow a simulated scenario in which students practice written and verbal communication, a clinical shadowing experience in which students observe providers communicating, or even a classroom-based discussion about sample written or verbal communication scenarios. Students could consider:

- The limitations in different forms of communication and the "intuition silences," where students' or other providers' sense of the situation failed to come through in conversation or documentation

- The strategies they or others use to navigate these limitations and alternative approaches

- Possibilities for change within medical communication practices to better accommodate intuitive information

As we develop this project further, designing lesson plans and activities to scaffold awareness and incorporation of intuition into healthcare communication pedagogy will be a primary priority. In addition, continuing to theorize the relationship between intuition, embodied experience, and patient sense is a focus of this ongoing project, as well as better understanding the similarities and differences in how intuition manifests at each stage of patient care. In doing so, this project aims to support healthcare providers and educators in better understanding their communication practices and valuing the intangible forces that guide these practices.

\section{ACKNOWLEDGEMENTS}

The EMS research project was funded in part by grants from the Purdue University Research Foundation and Towson University's Faculty Development and Research Committee.

\section{REFERENCES}

[1] L. A. Green, "What alters physicians' decisions to admit to the coronary care unit?," J. of Family Practice, vol. 45, no. 3, pp. 219-226, 1997.
[2] L.A. Green and F. J. Yates, "Influence of pseudodiagnostic information on the evaluation of Ischemic Heart Disease," Ann. of Emergency Medicine, vol. 25, no. 4, pp. 451-457, 1995.

[3] J. Groopman, How Doctors Think. New York: Houghton Mifflin, 2007.

[4] G. Klein, Sources of Power: How People Make Decisions. Cambridge, Mass.: MIT Press, 1999, p. 29.

[5] E. L. Angeli, "Three types of memory in emergency medical services communication," Written Commun., vol. 32, no. 1, pp. 3-38, 2015.

[6] J. Cushman, “'Write Me a Better Story': Writing stories as a diagnostic and repair practice for automotive technicians," J. of Tech. Writing and Commun., vol. 45, no. 2, pp. 189-208, 2015.

[7] L. Flower, "The role of task representation in reading-towrite," in Reading-to-Write: Exploring a Cognitive and Social Process. New York: Oxford, 2002, pp. 35-75.

[8] K. P. O'Hara, A. Taylor, W. Newman, and A. J. Sellen, "Understanding the materiality of writing from multiple sources," Intl. J. of Human-computer Studies, vol. 56, no. 3, pp. 269-305, 2002.

[9] A. Glöckner and C. Witteman, Foundations for Tracing Intuition: Challenges and Methods, Psychology Press, 2009.

[10] G. Klein, Sources of Power: How People Make Decisions. Cambridge, Mass.: MIT Press, 1999.

[11] B. A. Sauer, The Rhetoric of Risk: Technical Documentation in Hazardous Environments, New York: Taylor \& Francis, 2003, p. 134.

[12] B. A. Sauer, The Rhetoric of Risk: Technical Documentation in Hazardous Environments, New York: Taylor \& Francis, 2003, p. 159.

[13] B. A. Sauer, The Rhetoric of Risk: Technical Documentation in Hazardous Environments, New York: Taylor \& Francis, 2003, p. 134.

[14] C. Goodwin, "Professional vision," Amer. Anthropologist, vol. 96 , no. 3 , pp. 606-633, 1994, p. 606.

[15] C. Goodwin, "Professional vision," Amer. Anthropologist, vol. 96 , no. 3 , pp. $606-633$, 1994 , p. 606.

[16] J. Corbin, \& A. Strauss, Basics of qualitative research: Techniques and procedures for developing grounded theory $\left(3^{\text {rd }}\right.$ ed.). Los Angeles: SAGE, 2008.

[17] T. R. Lindlof \& B. C. Taylor, Qualitative communication research methods ( $2^{\text {nd }}$ ed.). Los Angeles, SAGE: 2002.

[18] L. R. Goldberg, G. R. Brown, V. A. Mosack, \& P. A. Fletcher, "Student reflections following exposure to a casebased interprofessional learning experience: Preliminary 
findings," J. of Interprofessional Care, vol. 29, no. 4, pp. 380$382,2015$.

[19] L. Montagna, C. Benaglio, \& L. Zannini, "Reflective writing in nursing education: Background, experiences and methods," Assistenza infermieristica e ricerca: AIR, vol. 29, no. 3, pp. 140-152, 2009.

[20] S. Epp, "The value of reflective journaling in undergraduate nursing education: A literature review," Int. J. of Nursing Studies, vol. 45, no. 9, pp. 1379-1388, 2008.

\section{ABOUT THE Authors}

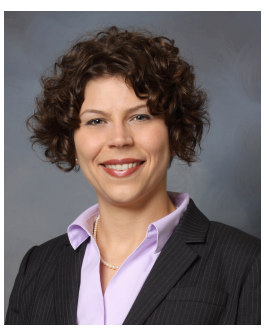

Elizabeth L. Angeli is Assistant Professor of English at Marquette University where she teaches courses in writing and rhetoric. As a rhetorician of health and medicine and technical communication researcher, she studies how healthcare professionals manage changing, urgent medical information in unstable medical contexts, including emergency medical services and public health crisis response. Elizabeth's work has appeared in Written Communication, Journal of Technical Writing and Communication, and Computers and Composition Online.

Lillian Campbell is Assistant Professor of English at Marquette University. Her research interests include the rhetorics of health and medicine, feminist rhetoric, writing in the disciplines, and technical communication. Her current research examines how classroom clinical nursing simulations initiate students into the writing, talk, and action of the nursing field. Aims include theorizing a rhetoric of patient simulation, modeling a multi-modal methodology for coding video data, and considering the implications of these findings for technical and professional communication pedagogy. Her work is published or forthcoming in Women's Studies in

Communication, Composition Forum, and Written

Communication.

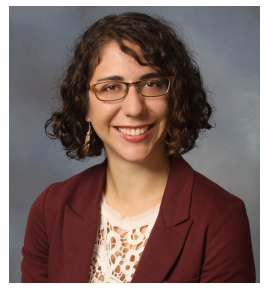

